\title{
Assessing the benefits and harms of low-dose computed tomography screening for lung cancer
}

\author{
Paul F Pinsky* \\ "Division of Cancer Prevention, National Cancer Institute, 9609 Medical Center Drive, Room \\ 5E108, Bethesda, MD 20892, USA; Tel.: +1 240276 7014; pp4f@nih.gov
}

\section{Summary}

The concept of using low-dose computed tomography (LDCT) for lung cancer screening goes back almost 25 years. In 2011, the National Lung Screening Trial (NLST) reported that LDCT screening significantly reduced mortality from lung cancer in a high risk population. This article evaluates the benefits and harms of LDCT screening, based largely on evidence from randomized trials. Harms include false-positive screens and resultant diagnostic procedures, overdiagnosed cancers, and radiation exposure. Benefits can be expressed as the number needed to be screened to prevent one lung cancer death or as estimated overall reductions in lung cancer mortality assuming LDCT population screening as recommended by guidelines. Indirect metrics of benefit, such as lung cancer survival and stage distribution, as well as measures of harms, will be important to monitor in the future as LDCT screening disseminates in the population.

\section{Keywords}

benefits; harms; low-dose CT; lung cancer; screening

\begin{abstract}
The idea of using low-dose computed tomography (LDCT) for lung cancer screening goes back almost 25 years [1]. LDCT was initially shown to detect more noncalcified lung nodules and more lung cancers than chest radiography [2-4]. The accumulating evidence regarding the potential of LDCT screening to detect lung cancer early and possibly prevent lung cancer deaths eventually led to the initiation of the National Lung Screening Trial (NLST) in the USA and other randomized trials in Europe [5-8]. In 2011, the NLST reported that LDCT screening significantly reduced mortality from lung cancer in a highrisk population (at least 30 pack-years of smoking) [8]. Recently, the United States Preventive Services Task Force (USPSTF) gave a grade B recommendation for annual LDCT screening from 55 to 80 years of age for persons meeting the NLST smoking eligibility criteria, stating that "annual screening for lung cancer with LDCT is of moderate net benefit in asymptomatic persons who are at high risk for lung cancer" [9]. The task force uses the term "net benefit" to reflect the overall balance of benefits versus harms of LDCT screening.
\end{abstract}

\footnotetext{
Financial \& competing interests disclosure

The author has no relevant affiliations or financial involvement with any organization or entity with a financial interest in or financial conflict with the subject matter or materials discussed in the manuscript.

No writing assistance was utilized in the production of this manuscript.
} 
Here, we review the benefits and harms of LDCT screening and discuss approaches to assessing the benefits-to-harms trade-off.

\section{Benefits of screening}

\section{Results of randomized control trials}

The NLST randomized almost 55,000 subjects aged 55-74 years to three annual rounds of screening with either LDCT or chest radiograph [8]. Eligibility criteria included 30-plus pack-years of smoking and current smoking or having quit smoking within the last 15 years. Overall, approximately $50 \%$ of NLST subjects were current smokers, and the mean packyears was 56. A noncalcified nodule $(\mathrm{NCN})$ of at least $4 \mathrm{~mm}$ in greatest diameter constituted a positive LDCT screen; other suspicious abnormalities (e.g., adenopathy) could also trigger a positive screen. Subjects were followed for a median of 6.5 years from randomization.

The NLST initially reported a statistically significant $20 \%$ reduction in lung cancer mortality for LDCT versus chest radiograph [8]. Subsequent publications examined a slightly longer follow-up period for lung cancer deaths and reported a $16 \%$ lung cancer mortality reduction, or a rate ratio (RR) of 0.84 (95\% CI: 0.75-0.95) [10]. Approximately 60\% of LDCT-arm lung cancers were screen detected, and of these, $62 \%$ were diagnosed in stage I.

Three smaller randomized controlled trials (RCTs) of high-risk current and former smokers - all in Europe - have also reported mortality outcomes [5-7]. Each offered five rounds of LDCT screening, and in contrast to NLST, compared LDCT screening with no screening. These RCTs reported RRs for lung cancer-specific mortality of 0.83 (95\% CI: 0.45-1.54), 1.37 (95\% CI: 0.63-2.97) and 1.99 (95\% CI: 0.80-4.96). The wide confidence intervals indicate the lack of precision of the RR estimates; combined, these studies only had $10 \%$ of the number of lung cancer deaths as NLST.

\section{Metrics of screening benefit}

In cancer screening RCTs, the generally accepted primary outcome is cancer-specific mortality, and the standard metric for measuring this is the RR cited above (i.e., the ratio of cancer-specific mortality rates in the two trial arms). From a public health perspective, a more informative measure of screening effectiveness is the number needed to screen (NNS), which is defined as the reciprocal of the difference in (cancer-specific) mortality rates between trial arms [11]. The NNS denotes the number of subjects needed to be screened in order to prevent one death from the cancer of interest. As such, and in contrast to the mortality RR, it is directly interpretable in terms of the required resources that need to be expended in order to obtain a given health benefit.

Although often left unstated, any NNS estimate refers to a given scenario involving the number and frequency of screens. In the NLST, where an NNS of 320 was computed, this estimate implicitly refers to three annual LDCT screens (i.e., 320 subjects need to be screened over three annual rounds [and followed for a total of 6.5 years] in order to prevent one lung cancer death) [10]. In addition, the study population from which the estimate was derived is also critical. In populations with lower risk than the NLST, the NNS would likely be greater since, assuming the same lung cancer mortality RR, the NNS increases as the 
population lung cancer risk decreases. Even within the NLST, former smokers had twice the NNS as current smokers (462 vs 230), due primarily to their lower risk level [10].

In terms of minimizing NNS, LDCT screening is favorable for several reasons. Although the percentage mortality benefit is modest at 16-20\% (corresponding to an RR of $0.80-0.84$ ), not only does lung cancer have a high overall mortality rate, but also a high-risk group can be easily identified and targeted for screening. A useful contrast is with mammography. In their respective intended screening populations, which for mammography is women aged 50-74 years based on the most recent USPSTF recommendations, the mortality rate from breast cancer is less than a fifth of the mortality rate from lung cancer for the LDCT recommended screening population. Because the percentage mortality reductions for their respective cancers for LDCT and mammography are similar, this translates into a NNS that is approximately five-times as large for mammography as for LDCT screening.

\section{Screening benefits in the population setting}

Since the NLST was conducted only over three rounds of screening and other LDCT trials or observational studies were also of limited duration, there is little direct evidence as to the long-term benefits (and harms) of continued screening in the population over the periods recommended by the USPSTF. For now, one has to rely on modeling efforts in order to estimate long-term effects. The Cancer Intervention and Surveillance Modeling Network (CISNET) lung consortium consists of five independent groups who developed microsimulation models for lung cancer natural history and screening [12]. All five groups modeled a number of LDCT screening scenarios for a hypothetical cohort of US subjects followed from 45 to 90 years of age. Based on the USPSTF screening guidelines and assuming $100 \%$ compliance, the CISNET models showed (on average) a 14\% population reduction in lung cancer mortality, with $19 \%$ of the entire cohort undergoing at least one round of LDCT screening [12]. As with any modeling exercise, these results must be viewed with caution, as they are based (largely) on extrapolating the findings from the three screening rounds of NLST to 25 rounds in the population setting. To this point, the variability of the five model predictions was wide, with a range of estimated population lung cancer mortality reductions of 4.8 to $23 \%$ [12].

\section{Indirect measures of screening efficacy}

In contrast to the direct mortality benefit metrics of screening efficacy estimable from a randomized screening trial as described above, a number of indirect measures of screening efficacy are widely utilized outside of the randomized trial setting. Two common such measures are survival statistics and stage distribution (i.e., comparing the survival and stage distribution of cancers detected under a screening program with those of cancers detected in a nonscreening environment). Each of these measures alone or in combination is not sufficient to conclude that screening has any mortality benefit, due to the widely known biases of lead time, length-biased sampling and overdiagnosis, and to the fact that a 'stage shift', or a more favorable stage distribution observed with screening, is a necessary but not sufficient condition for a mortality reduction [13]. Another metric of screening efficacy is test sensitivity, although again, high sensitivity does not necessarily translate into a mortality benefit of screening. 
Nevertheless, if there is already evidence of a mortality benefit from a well-conducted randomized trial, as there is with LDCT and lung cancer screening from the NLST, then these surrogate outcome measures may serve as important benchmarks for monitoring the performance of screening in clinical practice, since it is difficult for a variety of reasons (including self-selection of who chooses to be screened) to assess actual reductions in cancer-specific mortality outside of a randomized trial setting. In the NLST, 62\% of LDCTarm screen-detected cancers were stage I; furthermore, 59\% of all LDCT-arm cancers diagnosed during the screening phase of the trial were stage I [8]. LDCT test sensitivity was 93.7\%. All-cause 5-year survival of screen-detected cancers was 55\% for subjects over 65 years of age and $64 \%$ for subjects under 65 years of age [14].

\section{Harms of screening \\ False-positive screens}

One of the primary potential harms of any screening modality is false-positive tests, along with the resultant diagnostic work-up and any complications thereof. In LDCT screening, a wide range of false-positive rates have been reported, but almost all are on the high side for a screening test. Bach analyzed data from 11 cohort studies and eight RCTs; with one outlier excepted, false-positive rates (defined as 1 - specificity) ranged from 9 to $50 \%$, with a mean of $20 \%$ [15].

In the NLST, the false-positive rate for LDCT was approximately $27 \%$ in each of the first two rounds, decreasing to $16.8 \%$ in the third round [8]. The positive predictive values, defined as the proportion of positive screens with a subsequent confirmed lung cancer diagnosis, were $3.8,2.4$ and $5.2 \%$ in rounds $1-3$, respectively.

False-positive tests generate costs in terms of follow-up diagnostic procedures. In the NLST, 90.4\% of LDCT-arm subjects with a baseline positive screen received some diagnostic procedure, with $81 \%$ receiving imaging [8]. The proportion of positive screens receiving any follow-up procedures dropped to approximately $60 \%$ in the second and third rounds, with the proportion receiving imaging also dropping to $37-51 \%$. This decrease in diagnostic follow-up was due to the fact that, at later screening rounds, many of the positive screens involved nodules that were first identified at baseline and that were judged to be stable.

A relatively small proportion (2.7\%) of false-positive LDCT tests in the NLST were followed up with invasive diagnostic procedures (i.e., thoracotomy, thoracoscopy, mediastinoscopy, bronchoscopy or needle biopsy); the invasive procedure rate was higher for false positives at the baseline screen (3.7\%) than for those at subsequent screening rounds (2.3\%) [8]. Of the invasive procedures (following false-positive screens), 44 (9.6\%) had at least one complication, with 11 having a major complication. Among all LDCT screens, $0.6 \%$ had an invasive diagnostic procedure following a false-positive screen and $0.06 \%$ had a complication arising from an invasive procedure (for a false-positive screen).

An evaluation of the Danish Lung Cancer Screening Trial (DLCST) showed that mean annual healthcare expenditures were higher among subjects with false-positive screens (€4464) as compared with control-arm subjects (€2673) by a factor of 1.67 (95\% CI: 1.20 
2.32) [16]. Subjects with (true-) negative screens had similar expenditures to control subjects. Note that costs of the CT screening were excluded.

An important issue with respect to false-positive results concerns nodule stability. In the NLST, a nodule that was judged to be stable over 2 years (i.e., had not changed over three annual screens) could, at the discretion of the radiologist, be classified as a negative screen. This explains why the false-positive rate decreased substantially in the third screening round. In fact, this conversion to negative screens in the presence of (only) a stable nodule was not performed consistently. If all instances of stable nodules had been classified as negative screens, the false-positive rate at the final screen would have decreased to approximately $11 \%$ [17].

The importance of this for annual population screening is that after such screening reaches a steady state, the vast majority of screens would likely be repeat screens with a prior screen from 2 or more years earlier available for comparison. By contrast, only a third of screens in the NLST met such criteria. Therefore, with steady-state population screening, the (false-) positivity rate could be very substantially lower than the overall $23 \%$ rate seen in the NLST.

Another approach to lowering the false-positive rate is increasing the size cutoff for an NCN to constitute a positive screen. Henschke et al. showed that increasing the size threshold for positivity from 5 to $8 \mathrm{~mm}$ in NCN mean diameter (average of length and width) decreased the screen positivity rate from 16 to $5.1 \%$, with only a small percentage of screen-detected cancers $(6 \%)$ missed due to the larger cutoff size [18]. Similarly, data from the NLST showed that increasing the size cutoff (greatest diameter) from 4 to $8 \mathrm{~mm}$ decreased the false-positive rate from $23.5 \%$ to $8.0 \%$, with only a relatively small concomitant decrease in sensitivity, from 93.1 to $83.2 \%$ [19]. These decreases in the positivity rate are theoretical, based on retrospective analyses of data based on lower cutoffs. Whether screening in clinical practice, with real-world constraints, including defensive medicine, can achieve these low positivity levels (and if so, also retain high sensitivity) remains to be seen. Based in part on the Henschke et al. findings cited above, the National Comprehensive Cancer Network's (NCCN) current recommendations define a positive baseline screen as a $\mathrm{NCN}$ of at least 6 $\mathrm{mm}$ in mean diameter [20].

False-positive rates are typically reported per round of screening. However, with repeated screening, the cumulative false-positive rate, defined as the probability of receiving at least one false-positive result over the course of screening, is also of interest. In the ITALUNG trial, over four rounds of LDCT screening, the cumulative false-positive rate was approximately $50.0 \%$, while in the NLST (three rounds), the cumulative false-positive rate was $38 \%$ [8,21]. With recommended annual population screening over the 55-80 years of age range, the cumulative false-positive rate would likely be substantially higher than these estimates.

In addition to the financial and resource-utilization costs of diagnostic follow-up procedures and the possible medical complications from them, as well as the extra ionizing radiation from diagnostic imaging, false-positive results may also generate patient anxiety. In the NELSON trial of LDCT screening in Europe, Van Den Bergh et al. showed that participants 
with an indeterminate result (a positive screen with a recommended follow-up CT at 3 months) experienced increased lung cancer-specific distress in the short term [22]. Byrne et al. found that an indeterminate lung CT screening result increased state anxiety in subjects, although the anxiety then decreased over time [23].

\section{Overdiagnosis}

Overdiagnosis denotes the concept of a cancer that is detected through screening but would never have become symptomatic or clinically diagnosed otherwise. Overdiagnosis is the critical concern and the most important harm of prostate-specific antigen-based prostate cancer screening, and it is becoming more recognized as a significant concern in mammography screening for breast cancer. Although it is considered to be much less of a concern with LDCT screening, such screening does generate some overdiagnosed cases. Quantitative definitions of overdiagnosis rates vary; in addition, there are multiple statistical methods for estimating overdiagnosis, even given the same definition. Therefore, care should be taken when comparing overdiagnosis rates across studies.

Utilizing NLST data, Patz et al. defined the overdiagnosis rate as the proportion of LDCT screen-detected cancers that were overdiagnosed (i.e., that in theory would never have been diagnosed in subjects' lifetimes absent LDCT screening) [24]. Based on a natural history model, the overdiagnosis rate was estimated at $11 \%$.

In the population setting, where only a fraction of persons actually undergo screening, the overdiagnosis rate can also be defined as the proportion of all diagnosed cancers (including those in nonscreened subjects) that are overdiagnosed. For this definition, the rate depends critically on the percentage of the population that is undergoing screening, as well as the frequency of screening. Based on the recommended USPSTF guidelines (55-80 years of age with $\geq 30$ pack-years of smoking and a current smoker or who quit smoking within 15 years), the population overdiagnosis rate, as estimated by the CISNET modeling groups, was 3.7\% [12].

Overdiagnosis can also be assessed indirectly by examining indicators of tumor aggressiveness, such as tumor volume doubling time. Veronesi et al. found that $25 \%$ of incident LDCT screen-detected cancers were slow growing or indolent, as defined by a volume doubling time of over 400 days [25]. Although not all of these tumors necessarily represent overdiagnosed cases, this reservoir of tumors with indolent characteristics again points to the potential of overdiagnosis with LDCT.

While overdiagnosis per se (i.e., being diagnosed with and having a label of lung cancer) is a significant issue in terms of anxiety and possible insurance or employment ramifications, overtreatment as a consequence of overdiagnosis has the potential for serious medical adverse events. As such, one possible mitigating factor for overdiagnosis is to limit or delay treatment, and some researchers have suggested a 'wait-and-see' approach for tumors with apparent indolent characteristics [25]. However, given the connotations of a lung cancer diagnosis, this could be difficult to recommend for patients and physicians. 


\section{Radiation risk}

Radiation risk models are generally derived from epidemiologic data, in which the radiation exposures are considerably greater than those seen in medical imaging. Therefore,

estimating the radiation risk from LDCT screening relies on extrapolation to lower radiation doses.

Based on the NLST LDCT screening settings, with an average estimated effective dose of $1.5 \mathrm{mSv}$ and annual screening of high-risk lung cancer subjects from 50 to 75 years of age, Brenner estimated lung cancer excess risk due to LDCT radiation of $0.23 \%$ for males and $0.85 \%$ for females [26]. Using similar assumptions of LDCT dose and annual lifetime screening, Frank et al. estimated an excess risk of $0.07 \%$ for males and $0.14 \%$ for females [27]. The difference in estimates was due in part to whether an additive or multiplicative model was used in order to combine background and radiation-induced lung cancer risk. For context, current smokers aged 55 years have an approximately $15 \%$ lifetime risk of future lung cancer. The CISNET modeling group estimated 24 radiation- related lung cancer deaths compared with 459 lung cancer deaths averted, with a ratio of LDCT-caused (by radiation) to LDCT-averted lung cancer deaths of approximately 1:20 [12].

\section{Incidental findings}

LDCT lung cancer screening may identify incidental findings unrelated to lung cancer, such as emphysema or coronary artery calcifications (CACs). In the baseline round of NLST, $10.2 \%$ of subjects had a negative (for lung cancer) screen but clinically significant incidental findings [28]. A Canadian LDCT screening study found that $19 \%$ of subjects had incidental findings, with $0.8 \%$ of the incidental findings being severe (meriting immediate attention) [29]. An earlier systematic review, which did not include the NLST or the Canadian study, found a range of incidental findings of 7-27\% over four studies [30]. Incidental findings may lead to imaging or other follow-up procedures and thus may increase medical care costs; medical harms from follow-up procedures are also possible.

Of course, there may also be some benefit in detecting incidental abnormalities. For example; a study nested in the Dutch-Belgian NELSON LDCT lung cancer screening trial of high-risk smokers showed that $\mathrm{CAC}$ as measured from the screening examinations was predictive of future all-cause mortality and cardiovascular events [31]. However, whether such CAC scoring is clinically useful in this population has not been ascertained. More research in the general area of non-lung cancer findings on LDCT is needed. The USPSTF concluded that the benefits-to-harms trade-off of incidental findings cannot be determined [9].

\section{Evaluating the benefits-to-harms trade-off}

Figure 1 shows a schematic of outcomes per 1000 subjects scheduled for three annual rounds of LDCT screening. This graphic helps illustrate the trade-off between harms and benefits of LDCT screening. Approximately 356 subjects (per 1000) will receive at least one false-positive screen, of whom 18 will have an invasive procedure as part of diagnostic follow-up. A total of 24 will have a screen-detected lung cancer, of which three will be overdiagnosed and three will result in a lung cancer death being averted. 
In order to evaluate the trade-offs of any medical intervention, one must first be able to reliably quantify both the benefits and the harms, which is often quite challenging. The next step is often more difficult - devising a method for weighing multiple types of outcomes, which are typically not measured on the same scale and often involve disparate events. How can increased anxiety be compared with lung cancer deaths averted? Medical resource utilization and costs also need to be considered.

A standard method of assessing the effectiveness of a medical intervention - and implicitly of weighing benefits versus harms - is cost-effectiveness analysis. Cost-effectiveness analyses often employ the metric of cost per quality-adjusted life-year (QALY), in which the QALY attempts to incorporate quality of life effects as well as longevity. Villanti et al. estimated cost per QALY in a cohort of high-risk subjects ( 230 pack-years) aged 50-64 years undergoing annual screening for 15 years [32]. Their base-case analysis yielded a cost:utility ratio of US $\$ 28,000$ per QALY. They then compared LDCT screening with other preventative interventions. Colonoscopy (US\$8552 per QALY) and cervical cancer screening with Pap smears (US\$18,662 per QALY) had more favorable cost:utility ratios, while biennial mammography had a less favorable cost:utility ratio (US\$53,000 per QALY). Note that colonoscopy and Pap smears both prevent cancers as well as detecting them early, in contrast to mammography and LDCT.

Cost-effectiveness estimates often vary widely, due to not only the underlying studies and/or models that quantify the various harms and benefits, but also due to variability in cost estimates, differences in what types of costs are included (e.g., lost productivity) and the choice of perspective (provider/patient vs societal). In addition, intangible effects, such as anxiety, are difficult to apply cost estimates to. Assessing quality of life effects, in order to estimate QALYs, is also inherently subjective and variable.

The USPSTF does not (and cannot according to its mandate) explicitly consider cost in making its recommendations. The USPSTF methodology is to attempt to accurately quantify benefits and harms and then perform a qualitative assessment of the trade-offs. The USPSTF statement cited the following harms of LDCT screening: false-positive results, incidental findings, overdiagnosis, radiation exposure and psychological distress [9]. The task force also relied on the CISNET modeling findings in order to help assess the benefits-versusharms trade-off in the long-term population screening setting.

\section{Conclusion \& future perspective}

The greatest unknown for LDCT lung cancer screening is how it will translate from the research setting into the routine-use population setting. Most of the evidence cited above comes from RCTs or other research settings. Experience with other technologies demonstrates that performance in clinical practice often falls short of that demonstrated in the research setting. Therefore, it is imperative that as LDCT screening begins to penetrate into clinical practice, registries should be set up that capture the entire spectrum of LDCT screening, from the prescreening phase - patient selection, communication and counseling through the LDCT screen and diagnostic follow-up (including repeat screening), to lung cancer diagnoses and long-term outcomes.

Lung Cancer Manag. Author manuscript; available in PMC 2015 November 27. 
Four questions stand out as being particularly critical. First, what are the characteristics of those choosing to be screened - is their average lung cancer risk level based on age and smoking history similar to that seen in the NLST? Second, what definitions of a positive screen are being utilized and is the use of these generally more stringent positive screen definitions resulting in substantially lower false-positive rates than those seen in the NLST? Third, is the rate of invasive procedures as diagnostic follow-up for screens that turn out to be falsely positive being kept as low as that seen in the NLST, and are complication rates from such procedures also low? Finally, are the benchmarks set in the NLST regarding surrogate measures of screening efficacy, including survival, stage distribution and LDCT test sensitivity, being met in the clinical practice setting?

\section{Acknowledgments}

This includes employment, consultancies, honoraria, stock ownership or options, expert testimony, grants or patents received or pending, or royalties.

\section{References}

Papers of special note have been highlighted as:

• of interest; $\bullet$ of considerable interest

1. Naidich P, Marshall CH, Gribbin C, Arams RS, McCauley DI. Low-dose CT of the lungs. preliminary observations. Radiology. 1990; 175:729-731. [PubMed: 2343122] •• Original paper on low-dose computed tomography (LDCT) and lung cancer screening.

2. Kaneko M, Eguchi K, Ohmatsu H, et al. Peripheral lung cancer: screening and early detection with low-dose spiral CT versus radiography. Radiology. 1996; 201:798-802. [PubMed: 8939234]

3. Sone S, Takashima S, Li F, et al. Mass screening for lung cancer with mobile spiral computed tomography scanner. Lancet. 1998; 351:1242-1245. [PubMed: 9643744]

4. Henschke CI, McCauley DJ, Yankelevitz D, et al. Early lung cancer action project: overall design and findings from baseline screening. Lancet. 1999; 354:99-105. [PubMed: 10408484] • Early large-scale study of LDCT lung cancer screening.

5. Saghir Z, Dirksen A, Ashraf H, et al. CT screening for lung cancer brings forward early disease. The randomized Danish Lung Cancer Screening Trial: status after five annual screening rounds with low-dose CT. Thorax. 2012; 67:296-301. [PubMed: 22286927]

6. Pastorino U, Rossi M, Rosato V, et al. Annual or biennial CT screening versus observation in heavy smokers: 5-year results of the MILD trial. Eur. J. Cancer Prev. 2012; 21:308-315. [PubMed: 22465911]

7. Infante M, Cavuto S, Lutman FR, et al. DANTE Study Group. A randomized study of lung cancer screening with spiral computed tomography: three year results from the DANTE trial. Am. J. Respir. Crit. Care Med. 2009; 180:445-453. [PubMed: 19520905]

8. National Lung Screening Trial Research Team. Reduced lung-cancer mortality with low-dose computed tomographic screening. N. Engl. J. Med. 2011; 365:395-409. [PubMed: 21714641] •• Presents the results of the largest randomized trial of LDCT lung cancer screening.

9. Moyer VA. Screening for lung cancer: U.S. Preventive Services Task Force Recommendation Statement. Ann. Intern. Med. 2014; 160:330-338. [PubMed: 24378917] •• Recommendation statement from the United States Preventive Services Task Force.

10. Pinsky PF, Church T, Izmirlian G, Kramer BS. The National Lung Screening Trial: results stratified by demographics, smoking history and lung cancer histology. Cancer. 2013; 119:39763983. [PubMed: 24037918]

11. Rembold CM. Number needed to screen: development of a statistic for disease screening. BMJ. 1998; 317:307-312. [PubMed: 9685274] 
12. De Koning HJ, Meza R, Plevritis S, et al. Benefits and harms of computed tomography lung cancer screening strategies: a comparative modeling study for the United States Preventive Services Task Force. Ann. Intern. Med. 2014; 160(5):311-320. [PubMed: 24379002] • Uses modeling in order to estimate the benefits and harms of long-term population screening.

13. Croswell JM, Ransohoff DF, Kramer BS. Principles of cancer screening: lessons from history and study design issues. Semin. Oncol. 2010; 37:202-215. [PubMed: 20709205]

14. Pinsky PF, Gierada DS, Hocking W, Patz EF, Kramer BS. National Lung Screening Trial Findings by age: medicare eligible versus under 65 population. Ann. Intern. Med. 2014; 161(9):627-633. [PubMed: 25199624]

15. Bach, P. Request for coverage of LDCT by Medicare. CMS MEDCAC meeting on lung cancer screening with low-dose computed tomography. Centers for Medicare and Medicaid Services. www.cms.gov.

16. Rasmussen JF, Siersma V, Pedersen JH, Heleno B, Saghir Z, Brodersen J. Healthcare costs in the Danish randomized controlled lung cancer CT-screening trial: a registry study. Lung Cancer. 2014; 83:347-355. [PubMed: 24418526]

17. Pinsky PF, Gierada D, Nath H, Kazerooni EA, Amorosa J. National Lung Screening Trial: variability in nodule detection rates in chest CT studies. Radiology. 2013; 268:865-873. [PubMed: 23592767]

18. Henschke CI, Yip R, Yankelevitz DF, Smith JP. Definition of a positive test result in computed tomography screening for lung cancer: a cohort study. Ann. Intern. Med. 2013; 158(4):246-252. [PubMed: 23420233]

19. Pinsky PF, Gierada D, Nath H, Kazerooni EA, Amorosa J. ROC curves for low-dose CT in the National Lung Screening Trial. J. Med. Screen. 2013; 20:165-168. [PubMed: 24009092]

20. National Comprehensive Cancer Network. NCCN guidelines for detection, prevention, and risk reduction. www.nccn.org.

21. Pegna AL, Picozzi G, Falaschi F, et al. Four year results of low-dose CT screening and nodule management in the ITALUNG trial. J. Thorac. Oncol. 2013; 8:866-875. [PubMed: 23612465]

22. Van Den Bergh KAM, Essink-Bot ML, Borsboom G, et al. Short-term health-related quality of life consequences in a lung cancer CT screening trial (NELSON). Br. J. Cancer. 2010; 102:27-34. [PubMed: 19935789]

23. Byrne MM, Weissfeld J, Roberts MS. Anxiety, fear of cancer, and perceived risk of cancer following lung cancer screening. Med. Decis. Making. 2008; 28:917-925. [PubMed: 18725404]

24. Patz E, Pinsky PF, Gatsonis C, et al. NLST Overdiagnosis Manuscript Writing Team. Overdiagnosis in low-dose computed tomography screening for lung cancer. JAMA Intern. Med. 2013; 174(2):269-274. [PubMed: 24322569]

25. Veronesi G, Maisonneuve P, Bellomi M, et al. Estimating overdiagnosis in low-dose computed tomography screening for lung cancer. Ann. Intern. Med. 2012; 157:776-784. [PubMed: 23208167]

26. Brenner DJ. Radiation risks potentially associated with low-dose CT screening of adult smokers for lung cancer. Radiology. 2004; 231:440-445. [PubMed: 15128988]

27. Frank L, Christodoulou E, Kazerooni E. Radiation risk of lung cancer screening. Semin. Respir. Crit. Care Med. 2013; 34:738-747. [PubMed: 24258564]

28. The National Lung Screening Trial Research Team. Results of initial low-dose computed tomographic screening for lung cancer. N. Engl. J. Med. 2013; 368:1980-1991. [PubMed: 23697514]

29. Kucharczyk M, Menezes RJ, McGregor A, Paul NS, Roberts HC. Assessing the impact of incidental findings in a lung cancer screening study by using low-dose computed tomography. Can. Assoc. Radiol. J. 2011; 62:141-145. [PubMed: 20382501]

30. Jacobs PC, Mali WP, Grobbee DE, et al. Prevalence of incidental findings in computed tomographic screening of the chest: a systematic review. J. Comput. Assist. Tomogr. 2008; 32:214-221. [PubMed: 18379305] - Systematic review of incidental findings in LDCT lung cancer screening. 
31. Jacobs PC, Gondrie MJA, van der Graaf Y, et al. Coronary artery calcium can predict all-cause mortality and cardiovascular events on low-dose CT screening for lung cancer. Am. J. Roentgenol. 2012; 198:505-511. [PubMed: 22357989]

32. Villanti AC, Jiang Y, Abrams DB, Pyenson BS. A cost-utility analysis of lung cancer screening and the additional benefits of incorporating smoking cessation interventions. PLoS ONE. 2013; 8(8):e71379. [PubMed: 23940744] • Cost-effectiveness analysis of LDCT screening. 


\section{Practice points}

- Low-dose CT (LDCT) screening has been shown in the randomized National Lung Screening Trial (NLST) to reduce lung-cancer specific mortality in highrisk current and former smokers

- A harm of LDCT screening is the high false-positive rate and resultant diagnostic procedures; efforts are ongoing to reduce the false-positive rate without substantially increasing the missed cancer rate

- Due to the high mortality rate from lung cancer, and the ability to easily identify a high-risk population, LDCT screening potentially has a favorable costeffectiveness ratio (cost per quality adjusted life year gained)

- Monitoring of LDCT screening as it disseminates into routine clinical practice will be critical going forward to insure that it is being implemented in a safe and effective manner 


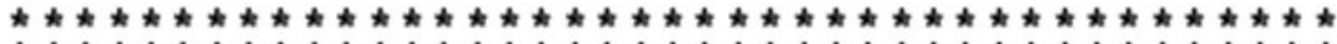

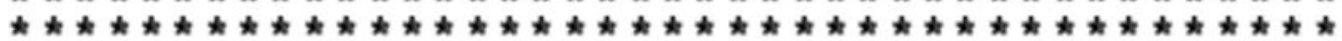

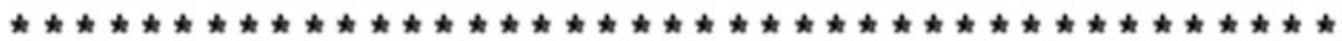

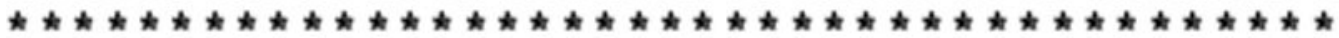

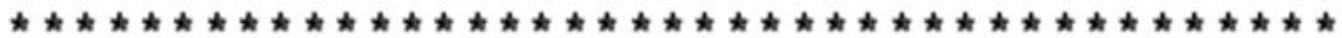

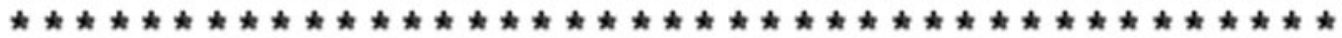

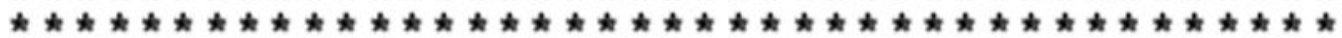

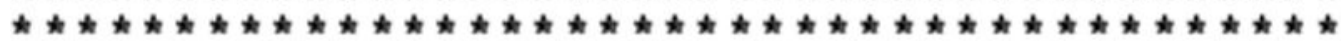

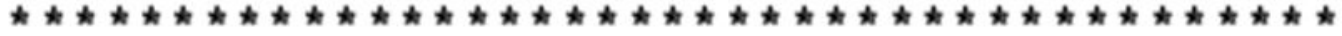

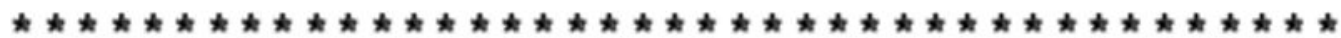

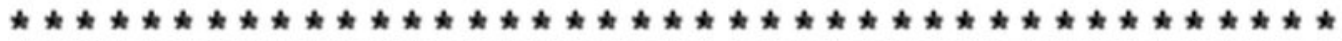

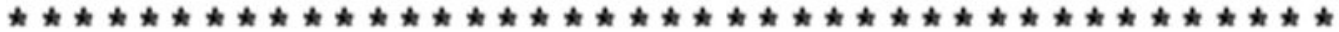

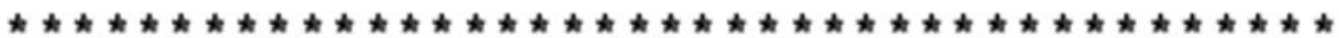

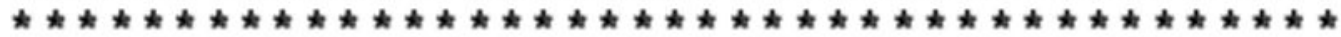

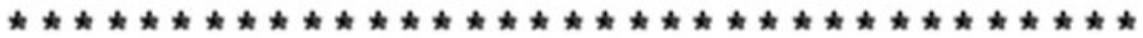

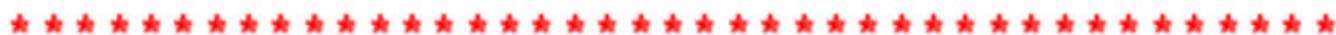

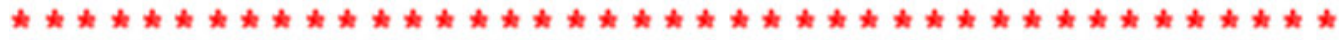

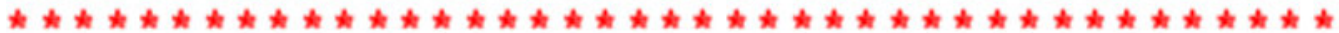

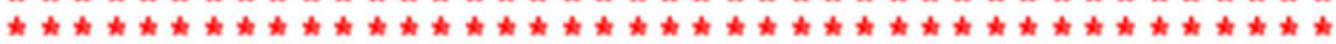

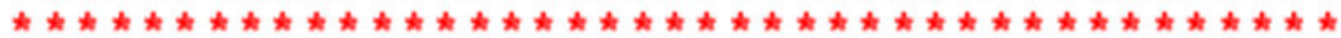

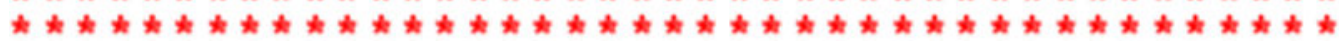

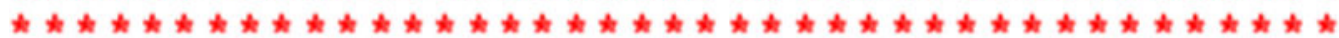

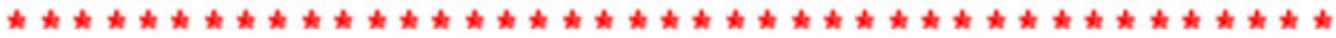

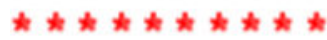

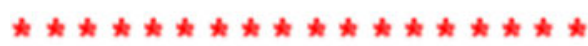

False Positive Screen/Invasive Procedure

$\star \star *$

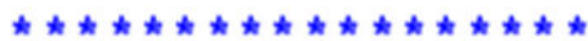

$\star \star *$

\section{Screen Detected Cancer/Overdiagnosed}

Screen Detected Cancer

Screen Detected Cancer/Lung Cancer Death Prevented

Figure 1. Schematic of benefits versus harms of low-dose CT screening

Outcomes for 1000 subjects scheduled for three annual rounds of low-dose CT screening.

Derived from the experience of the National Lung Screening Trial. Note "screen-detected cancer" row excludes screen-detected cancers that were overdiagnosed and screen-detected cancers where lung cancer death was prevented. Subjects with a false-positive screen and a subsequent screen-detected cancer at a later screening round are grouped with the screendetected cancers. 